\title{
Handheld Versus Wearable Interaction Design for Professionals
}

\author{
A Case Study of Hospital Service Work \\ Allan Stisen, Henrik Blunck, Mikkel Baun Kjærgaard, Kaj Grønbæk \\ Department of Computer Science \\ Aarhus University, Denmark \\ \{allans, blunck, mikkelbk, kgronbak\}@cs.au.dk
}

\begin{abstract}
With the blooming of new available wrist-worn devices there are potentials for these to support the work done in many professional domains. One such domain is hospital service work. This paper explores two wearable prototypes' challenges and opportunities to support future hospital service work. This explorative study was conducted with 4 experienced hospital orderlies who interacted with an application across two wearable concepts, and one handheld smartphone in five scenarios, not involving patients, in a hospital environment. The interactions were recorded with a chestmounted camera afterwards semi-structured interviews with each participant were conducted. This study shows that wearable computers can effectively support the maintenance work of the orderlies and has domain-specific advantages over the handheld smartphone, e.g., the former support glancing at the task information. Furthermore, we outline aspects to aid designers of next generation wearable designs for hospital service work.
\end{abstract}

\section{Categories and Subject Descriptors}

H.5.2 [User Interfaces]: Evaluation/methodology; Input devices and strategies

\section{General Terms}

Human Factors; Design.

\section{Keywords}

Wearable Computing; Handheld Computing; Mobile Work

\section{INTRODUCTION}

The professional domains in which wearable computing has been developed and evaluated are numerous. For instance, one of the earlier studies used a Head-Mounted Display (HMD) to support aircraft maintainers 25], by providing the needed information for the current task to the

Permission to make digital or hard copies of all or part of this work for personal or classroom use is granted without fee provided that copies are not made or distributed for profit or commercial advantage and that copies bear this notice and the full citation on the first page. To copy otherwise, to republish, to post on servers or to redistribute to lists, requires prior specific permission and/or a fee.

OZCHI'14, Dec 2-5, 2014, Sydney, Australia

Copyright 2014 ACM 978-1-4503-0653-9 ...\$15.00. maintainer. A more recent study has empirically compared four different picking prototypes in a simulated warehouse order-picking work situation 31]. Furthermore, the use of smart watches has been demonstrated as valuable in different applications to support maintenance work 6. While a wide range of evaluated wearable systems exists in different domains, there are few studies evaluating wearable technology to support hospital work [11, 29.

In the hospital setting, clinical work practices occur in a highly distributed information space 13 in which care is managed by arranging temporal $[22$ and spatial [3] coordination to provide continuous coverage of patient care. While most studies of hospital work have investigated the work of healthcare professionals (e.g. nurses and doctors), it has been noted 11, 29 that very few studies have focused on investigating the mobile hospital service work performed. The work performed by service workers such as orderlies is basically all the maintenance work necessary to support and sustain the clinical work of healthcare professionals and thus can influence the quality of patient care. As handheld and wearable devices have been used to support the cooperative mobile work in hospitals [5, 23, 26, 32], these technologies can offer several opportunities to also support the service work of hospital orderlies.

In this paper, we conduct an exploratory study of hospital service work to investigate possible interaction paradigms suitable for service workers. This study is part of a research project aimed at transforming the manual task planning of service personnel. This is done through migrating from a situation where a dispatcher with a phone and a stationary computer does the planning, to a new mobile and positionbased planning system that the service workers will use.

The empirical data of this study originates from interviews, observations and iterative prototyping. In particular, this paper reports the evaluation of two wearable concepts that are compared with a handheld smartphone-based concept in five scenarios.

In the following, we will present the related work on handheld and wearable devices as well as our study of hospital service work that emphasizes the main characteristics of service work. Next, we introduce a set of specific requirements within the hospital service domain and present a comparative evaluation of handheld and wearable devices. Finally, we present and discuss the results of our evaluation and highlight some implications for future design and implementation on wearable devices to support professionals such as hospital service work. 


\section{RELATED WORK}

While a wide range of evaluated wearable systems exists, there are few studies evaluating wearable technology support of hospital work. One example of such a study is concerned with the monitoring of patients, in an intensive care unit, and evaluated an HMD combined with a wristmounted keyboard 32. Their results show that the HMD can support the clinician's awareness of patients who are seriously ill. This will enable the clinician to be away from the specific hospital ward tending to other patients, and still immediately become aware when something serious happens at the hospital ward. Another example of a wearable system in a hospital context focuses on supporting hospital ward rounds 1. The study's focus was on enabling nurses and doctors with easy information access and sharing, while not hindering the normal workflow of the respective workers. Furthermore, another study, MACCS, has focused on providing nurses with a hands-free speech interface to manage their communication, which improved their workflow by improving the communication process 23. Another study has designed support for monitoring of nursing activities of medication 28. Moreover, preliminary work has been done in investigating how HMDs can support emergency medical teams by reducing the information complexity 33 .

Furthermore, several studies investigating mobile handheld technology supporting hospital workers exist. MobileWard is an example of such a study; it centers around an application supporting mobile information access in a hospital ward, in a context-aware manner 26. Another study based on a real-world deployment of mobile technology is the study of AwareMedia which included a mobile phone, AwarePhone, and an ambient display 5. AwarePhone, was mainly concerned with supporting social awareness among clinicians, using contextual clues.

\section{FIELD STUDIES AT HOSPITALS}

The study presented here involves two hospitals (HO1 and $\mathrm{HO} 2$ ), which are part of a bigger project supporting both hospitals' logistic departments' work with a mobile workflow application. Even though the studies were conducted at two different hospitals, the work conducted by an orderly is similar at the two hospitals. In Table 1 the specific details of the different methods and contexts are depicted. The two hospitals were included to both ensure generalizability beyond the hospitals' individual organizations and HO1 was included to get pre-deployment insights, and $\mathrm{HO} 2$ would then provide post-deployment insights.

The methodology for the study was selected to understand the actual activities of the orderlies' work before and after the deployment of PosLogistics. Before PosLogistics was deployed at $\mathrm{HO} 2$, pre-deployment interviews were conducted at HO2. The pre-deployment interview questions were concerning how the orderlies collaborate, coordinate and prioritize their tasks. This was done to investigate the current work practices before the introduction of PosLogistics. Shortly after deployment of PosLogistics observations were conducted at $\mathrm{HO} 2$, and observations and predeployment interviews were conducted at HO1. The observations were conducted within the day-shift period at both hospitals (roughly from 07:30 AM to 15:30 PM). Since the hospitals were staffed by a limited number of people outside of this period, it was deemed too challenging for the orderlies

\begin{tabular}{|c|c|c|}
\hline Method Context & $\begin{array}{c}\text { Hospital } 1 \\
(\mathrm{HO} 1)\end{array}$ & $\begin{array}{c}\text { Hospital } 2 \\
(\mathrm{HO} 2)\end{array}$ \\
\hline $\begin{array}{l}\text { Pre-deployment interviews } \\
\text { formal/informal }\end{array}$ & $3 / 7$ & $12 / 3$ \\
\hline $\begin{array}{l}\text { Post-deployment interviews } \\
\text { formal/informal }\end{array}$ & 0 & $4 / 16$ \\
\hline $\begin{array}{l}\text { Observations (hours) } \\
\text { Pre- / Post deployment }\end{array}$ & 70 & 5 \\
\hline Deployment of PosLogistics & 0 & $>1$ Year \\
\hline $\begin{array}{l}\text { Wearable Evaluation } \\
\text { (participants) }\end{array}$ & 0 & 4 \\
\hline
\end{tabular}

Table 1: An overview of the methods used and in which hospital they were conducted and the number of participants

to be observed. Six months later post-deployment interviews were conducted at $\mathrm{HO} 2$. In general, the post-deployment interviews covered how the current work practices, e.g., communication and coordination between the orderlies, changed after the deployment of PosLogistics. Since patient sensitive information could potentially be recorded, the informal interviews were not recorded, but extensive notes were taken. The formal interviews were all recorded and later transcribed. Both the pre- and post deployment interviews were semi-structured interviews, which on the one hand allowed a specific focus and on the other hand exploring other areas that could be important for the domain analysis.

\subsection{Interview guide}

The interview guide for pre-deployment interviews covered the following overall themes: task types, e.g., they were asked to describe the different types of work tasks they perform, coordination, e.g., they were asked to explain how and when they hand over tasks or contact co-workers for help. Lastly, the interviews also covered their overview with regards to both their co-workers and the tasks, how they get such an overview today, how they perceive the importance of it in their work and how they utilize such an overview. The post-deployment interviews covered the same overall themes, but were also extended with questions regarding the PosLogistics application, and they were asked if the application had improved some of the aspects. Furthermore, they were asked if the application had triggered changes in their work and their routines and if so what relations the changes had with respect to supporting their work.

\subsection{Analysis of empirical data}

Field notes from the observations and interviews were transcribed in extended text afterwards, and the recorded interviews were transcribed as well. The transcribed text from the observations and the interviews were separately analyzed through thematic analysis 7 . Thematic analysis was applied to extract emerging themes for each method (interviews and observations), to structure and codify themes. These themes were then merged together across the methods applied and then used to distil some of the key aspects of the work of a hospital orderly. Then these themes were formulated into design requirements for future interactive devices for the hospital orderlies.

\subsection{The hospital logistics and PosLogistics}

PosLogistics, a handheld mobile application for task management was designed as part of a research project, for sup- 


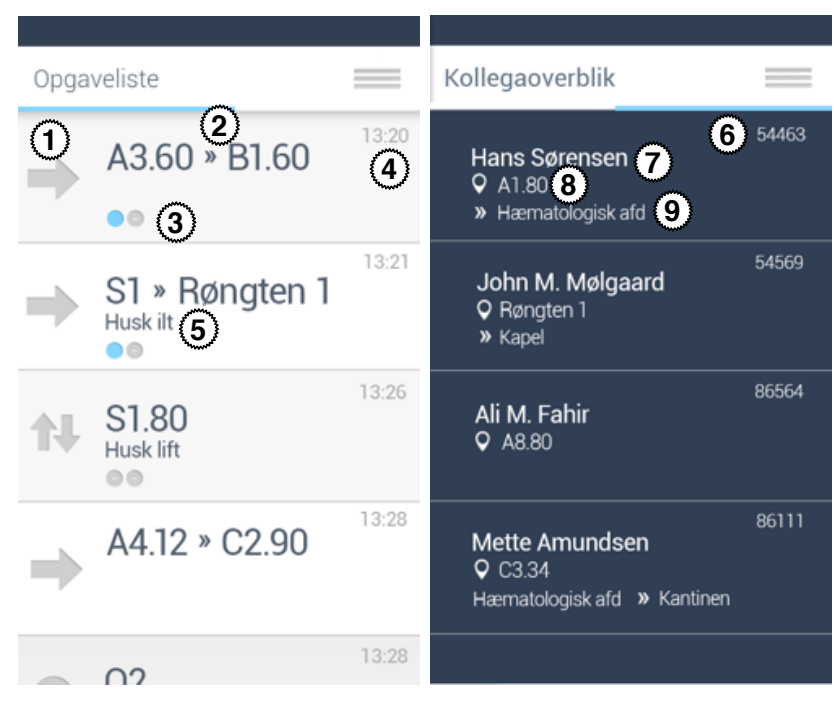

Figure 1: Screenshot of PosLogistics, on the left is the Task Overview and on the right is the Colleague Overview

porting the orderlies in maintaining an awareness of their tasks and their co-workers. The aims of PosLogistics are to support the orderlies' work both in enhancing the efficiency of their coordination of tasks and in reducing potential errors. PosLogistics has been designed by an external software company through interviews, observations, workshops and iterative prototyping sessions together with the orderlies and real-world evaluations. PosLogistics has two main views, both shown in Figure 1.

Task Overview: Shows all tasks across the hospital, which are currently waiting to be assigned. The task details shown are: the type of task (1) (indicated by an icon, e.g., an arrow for transport), task destination(s) (2), in case the tasks require several workers - how many have booked the task (3), task deadline (4) and task comments (5), e.g., requires an oxygen container.

Colleague Overview: To coordinate more efficiently, PosLogistics displays all colleagues' internal phone number (6), name (7) and current location (8) and their current task (9). Additionally, the wards and other departments have a screen at the entrance displaying the department's specific tasks and their progress, so that they can coordinate their collaboration with the orderlies and for example prepare a patient before transportation.

PosLogistics shows the orderly's co-workers' locations using Wi-Fi based indoor positioning, for details concerning specific positioning implementations and issues see 16,20 . Currently, PosLogistics is deployed at $\mathrm{HO} 2$ as a pilot project, while HO1 awaits the solution of some setup issues.

\section{HOSPITAL SERVICE WORK}

Based on the field studies presented in the previous section, the following section will present a work-domain analysis. A hospital orderly is responsible for maintaining basic, but important, functions at the hospital, so that the clinical personnel, e.g., doctors or nurses, can focus on their primary tasks. The orderlies' work primarily consists of practical, service based and heavy lifting tasks which are spatially distributed across the entire hospital. The tasks are generally characterized by the following four parameters, which are crucial for the interactions investigated in this study.

\subsection{Service tasks}

The tasks can either involve patients or maintenance service work. Tasks involving patients are transportations or mobilizations, i.e., transporting the patient between departments, or moving the patient out of the bed and into for example a wheelchair. These tasks also involve patient communication, since the patient can be stressed or in pain. $\mathrm{Pa}$ tient communication is required also because in order to ensure the safety of the patient and the orderly, it is important to instruct the patient about what to do and what not to do. In addition, it is also vital that the orderly's devices do not distract the patient or the orderly since this could cause both further stress. Moreover, it is generally perceived as rude to explicitly move the focus from the patient to a device, and this could break the patient's trust in the orderly. Furthermore, near-patient tasks require certain procedures to be followed. For example, orderlies should protect the patient against infections by wearing gloves. Some departments have further restrictions, e.g., the intensive care unit (ICU) has lot of tubes and monitors surrounding the patient, which should not be interfered with. Maintenance tasks are focused on keeping the hospital infrastructure and equipment functioning. This includes the following tasks: sorting and transporting trash from the wards, delivering goods, e.g., mail, or equipment, e.g., oxygen containers, and replacing equipment at the wards, e.g., replacing bulbs in certain scanners.

\subsection{Emergency tasks}

Second, the patient-related tasks can be acute, thus requiring the immediate attention of the orderly. For example, it is the orderly's role to manually perform CPR (CardioPulmonary Resuscitation), until the medical staff can take over with their CPR devices. Other examples include patient transports to acute operations within a short time span or assisting clinical personnel with patients with a problematic or violent behavior.

\subsection{High Mobility on Foot or on Vehicles}

It is a core characteristic of an orderly to be highly mobile and moving all over the hospital throughout the workday. To be more efficient they use electric trucks, busses, scooters, bed-pushers and bikes to transport themselves or patients across the hospital.

\subsection{Ad-hoc Coordination}

In order to render their work as efficient as possible it is crucial for the orderlies to coordinate with each other and the clinical personnel. Otherwise, the clinical personnel will have to either wait for the orderly, or perform the task themselves, which will waste valuable time for the nurses or doctors, e.g., if an operation is scheduled but the orderly fails to deliver the patient on time.

As an orderly's service-task plan is based on the departments' schedules, when they reschedule the orderly has to reschedule accordingly. Moreover, since a lot of the operations in a hospital do not follow their schedule, a study has shown only $18 \%$ of all operations follow their schedule 4]. The orderlies have to ad-hoc coordinate the tasks to stay effecient. This is done by coordinating the tasks and collaborating around their individual schedules, e.g., before PosLogistics by calling their co-workers and asking if they can take over a task, or know a nearby available colleague. 
Often the orderly knows some of the co-workers' routines and roughly understand their colleagues' work loads at specific times. This is often because many of the hospital's clinical departments have certain rhythms 22, depending on their type of medical work. This tacit knowledge enables the orderly to infer which co-worker is likely to be available. However, these rhythms are dynamic and emergencies or other situations may occur, which changes these at specific departments or the whole hospital.

PosLogistics augments the orderly's ability to infer about their co-workers' contexts to a large extent by showing the co-workers' current tasks (which includes the type and departments involved in the tasks) and their locations. Thus, the orderly can to a large extent infer the task progress of his co-workers, e.g, by looking at how close the co-worker is to the end destination. This inference can potentially limit the orderly's number of calls to his co-workers by selecting those from the colleague overview that seems suitable. Thus, having an awareness of their colleagues is an advantage for the orderlies to schedule tasks efficiently. This ensures that the patients show up on time and limits the clinical personnel's waiting time. Based on these characteristics the next section will describe requirements for future wearable devices.

\section{SPECIFIC REQUIREMENTS WITHIN THE DOMAIN}

From the work tasks as described in the previous section the following requirements with regards to wearable or mobile devices to be integrated in the work, arise:

On the move: The orderlies are highly mobile therefore it is necessary to be able to interact while being on the move, which also includes driving vehicles. It will lower the efficiency if the orderlies have to stop their movement to interact with their device.

Patient contact: This is another aspect of the work at a hospital, which imposes certain challenges and requirements on the devices, used in this context, e.g. managing interruptions, since interruptions should be avoided or at least limited in certain situations, as previous studies have confirmed 24. Situations include, for example, the orderlies' task of presenting dead patients to their relatives where the current practice for orderlies is to switch to silent mode. However, if the orderly forgets to switch back, this may result in missed notifications. Furthermore, when an orderly has close intrusive patient contact and instructs the patient on a procedure, a loud sound from a device can interfere with the instructions or stress the patient who is already in a stressful situation. This is highlighted from one of the interviews of an orderly:

(...) if you are in the middle of instructing a patient about moving the patient (...) If you are then being interrupted by a loud sound or similar. That is unpleasant. (O2)

However, it is not only stressful for the patients, but the orderlies can be stressed too, if they are not able to identify what the device attempts to communicate and how relevant it is. One of the orderlies elaborates on how it can remove their focus from the task, which can be dangerous for the patient:

(...) if you can feel that it is vibrating or beeping (sound) to show that a task has arrived. How important is it? (...) that is a stress factor (...) and it removes the focus from what you are doing. Because your thoughts are suddenly down in the pocket with the phone (O1)

Patient safety: Another issue raised by the orderlies was that the patients should feel safe when at the hospital. For example, they should not be afraid of any unnecessary harm coming to them, e.g., scratches or bruising from the orderlies lifting the patient. Additionally, the patients should not worry about their privacy. Thus a device should not broadcast patient-sensitive information to unintended persons. Furthermore, cameras in general are prohibited in patient areas. This is strictly enforced even if the camera is turned off when patients are nearby.

\subsection{Drawbacks with handheld devices}

Based on existing studies' data, the following issues are known with handheld devices:

Information Access time: Since the handheld phone is in a pocket or similar when it is not used, it takes $66 \%$ longer to access the phone compared to a phone on the wrist 2], and on an average $78 \%$ of the time of conducting a phone interaction is spent on picking up the phone from the pocket 2 .

Out of view: Another issue arising from having the phone in the pocket is that the screen is not viewable in all situations. To overcome this issue most phones have multimodal feedbacks, e.g., haptic and audio when delivering notifications. However, this can introduce attention overload to not only the owner of the phone but also to people nearby 14. Furthermore, sound can in certain situations be perceived as rude since it by nature is more intrusive and thus disturbs people around the user 18. To address this issue users often turn their device to silent mode [18, which introduces the risk of missing a notification. Extending upon this, the following issues were discovered as part of the empirical data of this study:

Notifications are missable: When the handheld smartphone is in a silent mode; the default choice, haptic feedback, is in some situations not enough to be noticeable. Thus, the user can miss an important notification or information:

There are many who would put it (smartphone) in silent mode. That is a problem (...) because some relevant information that is more important (...) could arrive (O1) This is especially important since notifications at hospitals can be emergency tasks, where a patient's life is at stake, which could be missed.

Hand(s) are required for interaction: As orderlies use their hands for practical tasks, e.g., turning or moving a patient, it is not always possible to have a hand free to hold a phone. This means that the orderly cannot interact with the phone, and should postpone the interaction until it is possible. Moreover, phones used daily by health care workers are a source of bacterial contamination 30.

Consumer devices as work tools: Using a phone at a work place may lead to unintentional messages and misinterpretations by, e.g., patients and co-workers. Thus, these consumer devices need to be adapted to the work environment. As this quote from one of the orderlies shows:

When I am at the departments and use a phone I always have it at the back of my mind that patients and their relatives will think: "that guy there is he just playing with a phone instead of working?" (...) People have to be able to see that it is not only a phone. (...) It is a work phone. (O3) 
Patients and colleagues can perceive interaction with a consumer device as a personal activity even though it is work related - since from a distance only the device's form factors, but not what the worker is doing. This introduces challenges to the orderlies who have to plan their usage of these devices in order to not be perceived as doing non-work related activities.

Hygiene: Hospital-acquired infections are a general concern at hospitals. To reduce the occurrence of these infections a Bare Below Elbows (BBE) policy has been strictly enforced in many countries, thus workers in the proximity of patients are not allowed to wear anything below the elbows. The BBE policy hinders certain wrist worn wearable devices to be directly adopted, or requires certain special designs of such devices.

Emergency situations: An orderly's task can be acute, e.g., performing CPR, or a patient transport to urgent operation. Thus, a device should either support these situations or be ensured not to interfere with the task.

Heavy lifting tasks: The orderlies perform heavy lifting tasks with patients and equipment. Thus, any device used should not hinder or complicate these tasks, e.g., by causing harm to the patient or scratching the skin or interfering with inserted tubes. In light of these requirements the next section will describe the evaluation of the proposed wearable devices.

\section{COMPARATIVE EVALUATION}

Based on an analysis of the orderlies' work (see section 4) and the drawbacks of the handheld devices (see subsection 5.1), a comparative study, which investigates the potential benefits and issues with a handheld smartphone and two wearable devices was conducted. Our goal was to investigate the potentials the forearm-placed wearable devices could have on hospital logistics work, and not to provide a statistical breakdown of the uses of the wearable prototypes and the mobile device.

\subsection{Evaluation setting}

After PosLogistics had been deployed at HO2 for roughly 10 months, the following comparative evaluation was conducted at HO2. HO2's orderlies were chosen to evaluate the wearable prototypes because of their experiences with using PosLogistics in their work. Based on the acquired empirical data from both interviews and observations, 5 representative scenarios were created. Each scenario was described to the orderly and the prototypes' screen would show sufficient information for the orderly to enact the scenarios. The participants in this study were 4 experienced orderlies (3 male and 1 female) in the age range of 25-50 years. Thus, the participants resembled the overall orderly population well, since it contains mostly males in varying ages. The 4 orderlies went through the 5 representative scenarios individually three times, with the three different devices (handheld smartphone, forearm-placed smartphone and a smartwatch all shown in Figure 2). Each orderly was instructed on how to use the specific prototype before the scenarios started and time was allowed to become comfortable with the prototype. Each participant was interviewed after the run-through of the 5 scenarios with the three devices. The interviews were semi-structured and covered the devices' interaction design and wearability focusing on their work in a hospital service setting and lasted between 30 and 75 minutes.
Interview guide: the interviews were constructed so that first the orderlies were asked about the devices with regards to each scenario overall, e.g., what device would you prefer and why, in the first scenario? Next, the interviews were followed by asking questions for each device with regards to: their perceived usability (with questions inspired by the SUS scale 8], the applicability in the domain, e.g. would there be any situations where you would not use the device?, the device's interaction modalities, e.g. touch-screen, audio and haptic feedback and the social acceptance of each device. Furthermore, for the wearable prototypes (see Figure $2 \mathrm{~b}$, Figure 2c the interviews covered the wearability of the device, with questions inspired by the questionnaire to assess wearability 17 .

While the participants were interacting with the devices, a chest-mounted GoPro camera was recording, and a screen recorder was running on the device. This setup was chosen to analyze the usage and interactions with the specific devices and to evaluate with regards to their perceived usefulness in this domain. Similar approaches of video recording have been used previously in the hospital domain 26] and generally within HCI 9]. The interaction with the devices were for the purpose of the analysis transcribed by describing how the user was interacting and holding the device, what had triggered the interaction and what was the intent. Furthermore, if the user just raised the wearable prototypes it was also transcribed for further analysis.

In order to be able to do the camera recording, the experiments' locations were constrained, because of the patients' privacy, to take place in areas at the hospital where patients rarely show up. The experiment location chosen was around and just outside the break room for the orderlies, which is placed in the basement next to the emergency ward. This also implied that we were restricted to scenarios that did not involve patients in any way, but mostly focused on the orderly-device interaction.

\subsection{Prototypes for evaluation}

The interface designs of the prototypes' interfaces are highly inspired by the already deployed PosLogistics' interface. This was done to enable the orderlies to draw upon their existing experiences with PosLogistics. An additional feature, switching between three different task-sorting settings in the task overview, was added to PosLogistics on all three devices. This feature was added to make the prototypes more comparable and the task planning easier and had been previously requested by the orderlies. In effect, all three devices were able to show the same information and details.

Handheld: A handheld smartphone (Samsung Galaxy S3 mini with a 4 inch touchscreen) was included in the study to make it easier for the orderlies to compare. In the task overview the handheld could show 8 tasks see Figure 2a

Smartwatch: A Samsung GalaxyGear smart watch (with a 1.6 inch touchscreen). With the limited screen space it could show 3.5 tasks in the task overview see Figure $2 \mathrm{~b}$

Forearm-placed smartphone: A Samsung Galaxy S3 mini placed on the forearm in landscape mode close to the elbow using a regular sports armband. The purpose of this prototype is to test another area closer to the elbow and investigate what potential a bigger screen size has in the chosen scenarios. This prototype was inspired by an earlier study on supporting maintenance work 10 . The device is shown in Figure 2c To fully utilize the landscape mode, the 


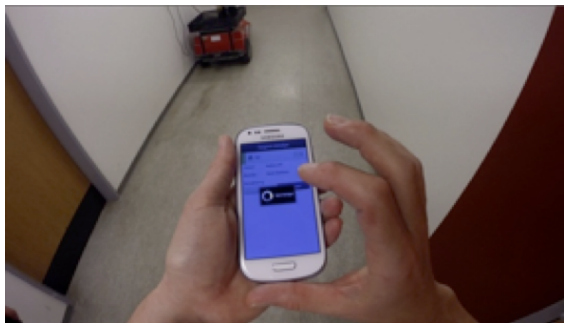

(a) Handheld smartphone

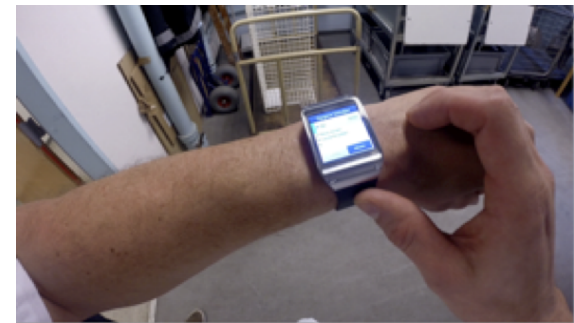

(b) GalaxyGear smartwatch

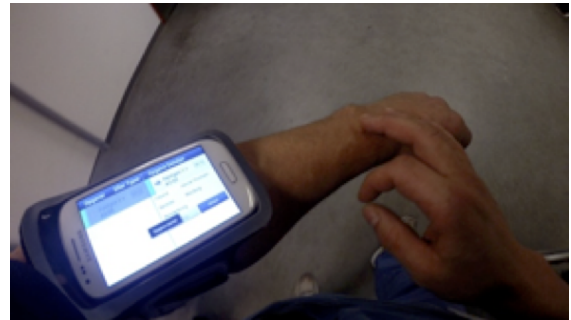

(c) Forearm-placed phone

Figure 2: The three prototypes used in the study as covered by the GoPro camera.

task overview was designed so that the task's details were right next to the task list. Whereas the other prototypes would show this in a new view. Thus, the user could, on a single screen, view both the task list and task details.

The wearable prototypes' placements were based on: the forearm as it is perceived as one of most unobtrusive areas 12, the most positive third-party perceived placement 21] and having the lowest reaction time based on visual alerts 15. However, the BBE policy described above could prohibit the use of the wearable prototypes. Based on the requirements presented in section 5 the two wearable prototypes presented are breaking the last requirement concerning the BBE policy. Nonetheless, it was the intention of this study to investigate potential interactions with a forearmplaced device. As the policy in the future could potentially recognize the introduction of new materials or effective procedures of device cleaning. Furthermore, not all orderlies are subject to the BBE policy because they have no patient contact.

The wearable prototypes utilized gesture recognition, using a similar approach as uWave 19], where the user could switch between different screens using gestures inspired by 1], and drop all their tasks which could be useful in emergency situations. However, the accuracy was not perceived as useful enough by the orderlies, thus it is not covered in the results section. Furthermore, to make the wearable prototypes simple and glanceable, the prototypes' screens were on all the time and dimmed after 30 seconds of inactivity.

\subsection{Scenarios}

In order to make the scenarios more realistic, a few imaginary department names were created around the evaluation setting. The five scenarios chosen to represent and evaluate the prototypes are the following:

S1 Overview: The orderly should choose the next optimal task to start in a queue of 10 given tasks.

S1 was chosen because the orderlies often have to prioritize their work schedule in an efficient manner. This means they have to prioritize and schedule their tasks quickly.

S2 Notification: The start condition is that there are no tasks in the queue and that the orderly walks to the break room. Shortly after the list contains a new tasks, and the user is notified. The user starts the task and walks towards the department. Later, a notification tells the user that the task is cancelled.

S2 is especially important as the overall plan at the hospital is dynamic and the orderlies have to be properly notified of important changes, e.g., the arrival of emergent tasks or cancellations to ad-hoc coordinate their tasks.
S3 Routine Task Switch: The user starts a transportation task and after having delivered the patient, the task should be filed as completed and a new task must be selected and started.

Furthermore, as the PosLogistics requires explicit interactions to update the orderly's work state it is important that it is efficient and easy to change the state of the work.

S4 Emergency Task: After having started a service task, suddenly an emergency task arrives in which the orderly quickly needs to accept the task and hand over his previous tasks and declare himself indisposed.

Since a hospital is a place where emergencies happen regularly, it is important that the devices can support these, so that the orderly can focus on his primary task instead of the interaction.

S5 At a glance: The user starts a patient transportation task and walks towards the department. When the orderly is close to the ward, the orderly has to check the name of the patient before the task can begin.

S5 is covering the ability to access information discreetly and easily, and the scenario is highly relevant since checking the patient name and Civil Registration Number is an important procedure to reduce errors at the hospital.

In the post-experiment interviews the orderlies were asked whether they found the scenarios representative of their work. They all gave affirmative answers, whereas one noted correctly that the scenarios did not cover any patient contact which was intentionally not part of the scenarios.

The transcribed data from the videos and after-scenario interview transcriptions were analyzed focusing on key themes, as part of the thematic analysis 7 . These themes then form the next section which presents the results of this study.

\section{RESULTS FROM EVALUATIONS}

Based on the video analysis of the orderlies executing the scenarios S1-S5 and the interviews afterwards, the following themes were identified. The quotes are used to describe themes and illustrate those across participants and data.

\subsection{Continuing with tasks}

In general the easy access to information with wearable prototypes was highlighted and perceived as beneficial to support the orderlies' work. The orderlies agreed that the closer the devices were to the hands, the easier it was to interact with, i.e., the wearable prototypes were easier to interact with, as this quote illustrates:

(...) where it is just easier that you don't have to take the phone out of the pocket (...) you have it right by your 


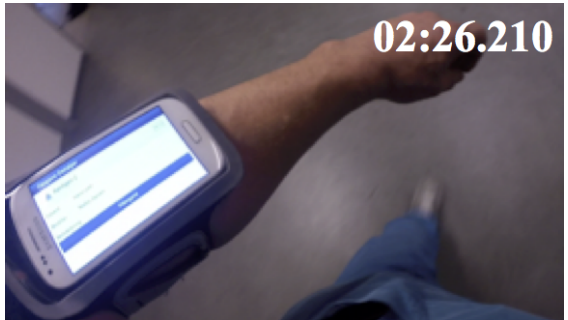

(a) It is noticed that the task is not started

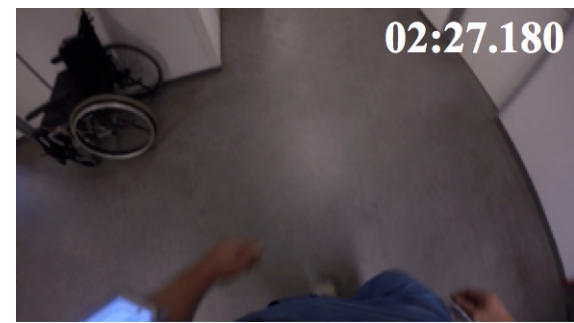

(b) The phone is lowered while walking

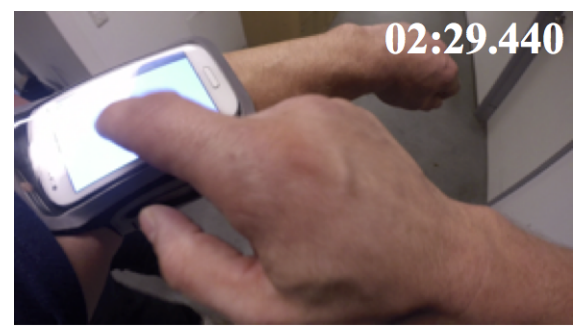

(c) The phone is raised to start the task

Figure 3: The orderly thinks he has started a task. However, at a glance he realizes that he has forgotten to start it.

hand, quite literally. (...) The closer it is to your hands, the easier it is. It almost becomes a reflex (...) you are almost started by having it right in front of you. (O1)

O1 focuses on the fact that the interaction with the wearable prototypes is perceived as being instant, e.g., when the wearable devices vibrates the user can instantly glance at the device to decide whether to initiate a interaction. Thus, the orderlies could with the fast information access interact on the go and easily switch from their task to PosLogistics and back again. This was perceived as the most valuable feature of the wearable devices for this work domain, which has already shown to be the dominant characteristic of wearable systems in the leisure domain 19, 27. However, the users did have some issues with the prototypes and their wearabilities, which the next section will present.

\subsection{Wearability in Hospital Service Work}

When designing wearable products it is important to consider several factors concerning the actual wearability of the product, e.g., placement, size, form etc. of the product 12]. Based on this, it is an important aspect of this study that the wearable prototypes should not interfere with the orderlies' work. The orderlies did in general agree that the wearable prototypes could be beneficial to support their maintenance work. However, one challenge is the close patient contact, and the safety of the patients. This means that the prototypes should in no way potentially harm the patients (presented in section 5). All the orderlies were concerned with this issue. One might propose that the orderlies could simply take off the devices when doing patient related tasks. However, this was not perceived as a satisfactory procedure.

A single orderly, O4, had difficulties with adjusting the sports band. As the band was not tightened enough it was constantly gliding down to the wrist. Naturally O4 was describing the phone on the arm setup as "dumb" or "awkward". During the video analysis it was also clear both of the wearable prototypes were adjusted several times both before and after the interactions. However, the smartphone on the arm was adjusted more frequently than the smartwatch and many of the orderlies used their thumb to stabilize the phone on the arm while touching the phone's display. Thus, the smartwatch was more stabile during the interactions. The interviews also supported this, where all the orderlies agreed on the smartwatch as being more comfortable to wear. O3 said that the band was not wide enough, and suggested to use a sleeve which could make the phone more stabile and comfortable to wear.

\subsection{Glanceable Awareness}

During the experiments, S2 and S4 were the only scenarios where the orderlies had to interact or look at the screens before returning to the imaginary departments. However, in other scenarios when the participants were walking between the imaginary departments to complete their tasks, three of the four orderlies brought the wearable prototypes into their view while walking, without any explicit intents, i.e., they were just glancing at the prototypes.

A particularly interesting situation occurred while one of the participants enacted S3, which confirms that the phone on the arm can support a peripheral awareness of task plans. As the orderly was finishing the first task, he forgot to start the second task and moved on. Later, he slightly adjusted the phone, Figure 3a, and lowered it again, Figure $3 \mathrm{~b}$ Shortly afterwards (3 seconds after the first glance), he raised the phone again, Figure $3 \mathrm{c}$ and says:

Look, I did not get the task started, but I can do that now (O2)

When immediately asked how he noticed it, he said that something caught his attention (though he was unsure what):

(...) I looked at the screen because I could feel that it was rolling and then I looked (...) There was something, which was shaking on my hand, (...) whatever happened I don't know (O2)

However, after careful analysis of videos and logs, the phone neither vibrated nor changed view in the situation. The last time it changed view was more than 6 seconds before the above situation. Furthermore, the orderly had already glanced after the view change before the glance shown in Figure 3(a). The only clues that were given to the orderly, in which he could notice that the task has not been correctly started, were two visual clues on the screen. First, a green bar showing the task had been started was missing, and second the button 'start' was active. These given clues were only perceived at a glance (less than a second) while walking. As most orderlies used similar strategies with the smartwatch, this can also support such awareness. All orderlies also agreed that easy access to information is important in their work, and that all the wearable prototypes supported this; especially in regards to supporting the maintenance work, as is exemplified in the following quote:

(...) it is an advantage that you can have both hands on the wheel and don't have to put the hand down in the (pocket) and check, check, check. (...) you just can raise the arm to see if it is urgent or what (O3)

$\mathrm{O} 3$, as the rest of the participants, perceived it as easier if the device was closer at hand. The interaction was perceived faster and better at reducing the time spent on doing the routine tasks, e.g., to start and finish a task. Based on these results, the prototypes presented in this study were 
perceived as being able to support peripheral awareness of the logistics tasks. Moreover, all the participants highlighted the ability to glance at the wearable displays as being at least as discrete or more than having to take out the phone. As earlier described this is especially important when the orderlies are in the proximity of patients.

Moreover, as it is necessary for the orderlies to have an overview of the unassigned tasks and their co-workers tasks, they emphasized that fast access to task information is important. They described how they often look at the tasks and the colleague overview, while doing their tasks:

That is because you regularly keep that overview of what tasks there are and how far the others are. So you follow up all the time on what is happening in the house. (O2)

When asked how they maintain the overview, and how they plan their task the orderlies were describing how they regularly check the task queue and their co-workers. The following quote is an example of an orderly describing how he is looking at the phone, without necessarily needing the information:

Yeah, and that also has something to do with whether I am going to end up there, there might be a task up there, where it would be efficient if I take instead of someone else. So that there is a flow. (...) If it is possible. It is good to have the overview, always. (O4)

After having described the orderlies' perception of the wearable prototypes potentially supporting the ability to glance and get an overview of the tasks; the next section will be concerned with the task overview and screen sizes.

\subsection{Screen size and Task Overview}

As the smartwatch could only show 3.5 tasks, which are fewer than the two other prototypes, it was expected that this could have a negative influence on the participants' perception of the smartwatch. Nevertheless, all participants did agree that the smartwatch's screen was adequate for this purpose. Two major features considered relevant for this were the ability to scroll through the list, and the employed task sorting. As explained by one of the orderlies when asked if it was easy enough to get an overview on the smartwatch:

Yes, because you have the ability to scroll up and down. That (the scrolling) is the same with the ones we have now. They can also show lot of tasks, (...) it is always those that are most current that are in the top of the screen. (O4)

O4 compares the scrolling on the smartwatch with the smartphone scrolling. The orderlies' comments towards the smartphone on the arm assessed the same strategies in a similar manner. This shows the importance of the task sorting when the orderlies need to schedule their plans, and that the currently-used strategy to a large extent supports this. Furthermore, the orderlies' task details consist of simple and precise descriptions, which make it easier to sort but also to present it in the user interface on all devices.

When the orderlies were asked if the smartwatch could be the only device used in their work setting, two of the orderlies were more skeptical. This was based on future plans to extend the handheld smartphone with other features besides task management. Based on this, the smartwatch was enough with regards to the current work practices and interaction design. However, for the smartwatch to support future applications and device usage, the smartwatch was perceived as being not enough. New interaction designs for the future work practices and device usages might enable the smartwatch to support such needs.

\subsection{Work tool and social perception}

An important aspect, social acceptability, which has been documented in general in the leisure domain, was also covered in the interview guide. One of the concerns of the orderly is how the patients perceive the interaction with a regular consumer smartphone. This showed that the orderlies have made several individual arrangements for interacting with the task management system, based on the potential social perception of both co-workers and patients. One of the most used examples is when checking a patient's name before transporting the patient. One of the orderlies describes his current procedure when being at the ward:

(...) I check the patient's name once more. (...) and I do that in front of the patient, and that is to show that "Okay I am not on facebook, I am looking up your name" and then I tilt the phone so that the patient can see a screen showing their name that does not look like a media page (O3)

Another orderly would turn away from the patient and then glance at the phone, by tilting it while it was in the pocket, to check the patient's name. Where other orderlies did this before entering the ward, so that the patient could not see the interaction.

When asked if the wearable devices will be socially acceptable to wear, on the one hand the orderlies described that the wearable prototypes would probably give them some extra attention or comments, which O1 mentions in the following quote:

I will have to accept some clever comments, but that is probably only until people gets used to it, but that is not an obstacle (O1)

Furthermore, before, while and after the orderlies were going through the scenarios they received comments from their coworkers with references, e.g., to RoboCop, and they received extra attention during the evaluation. Thus, the wearable devices could be perceived as not being applicable in this domain, due to this fact.

On the other hand, the orderlies, as the quote above shows, did not perceive this as a limitation of the wearable devices. Rather, the orderlies all agreed that the wearable prototypes looked more like a work tool. Thus, it would not be an obstacle to the applicability of the wearable devices:

(...) it is a good work tool, and the fact that it is strapped to the arm, I can't see that as a minus (...) There are other workgroups, such as doctors and nurses, who walk around with their stethoscope around the neck (...) this is a central work tool for our department. Which is why it should be as easy to use as possible (O1)

Furthermore, all the orderlies described, that the fact that they would obviously receive extra attention because of the wearable devices, was the same fact which caused the prototype to be perceived more as a work tool compared to e.g. a regular smartphone. As this quote highlights:

More than if you were walking with a regular smartphone, because then people can see: "Yeah, okay that is on the arm so it must be something work related" $(\mathrm{O} 2)$. 
Whereas the smartphone is easier to hide away which could be perceived as the device not being work related. All orderlies agreed that, if the wearable devices were an official work tool they would find it socially acceptable to wear them. The smartphone on the arm was perceived more as a work tool than the smartwatch. However, the orderlies also expected the smartphone on the arm to generate the most attention and comments. The next section will discuss the highlights from the results and potential impact on future reearch.

\section{DISCUSSION}

The data presented included investigations of highlights in mobile and wearable computing in a hospital setting where previous research has been limited to the clinical work. The investigation has shown that in the hospital service domain wearable computing has the potential to overcome the shortcomings of handheld devices. In general, the wearable prototypes' strongest advantage, is always being within reach to interact and view; but it is also one of its biggest issues. The devices presented in this study were lacking in the support of the patient related aspects, especially due to the BBE policy, whereas the maintenance support aspect was covered.

The potential for wearable support in hospital service work is that the information they require is, compared to clinical workers, less information rich. Thus, they do not need to reduce the information complexity in the way that earlier work has focused on 33. Rather, the information the orderlies need are concerned with acquiring an overview to enable effecient task planning. Furthermore, social awareness has also been investigated focusing on clinical work [5], since their work is often depending on certain specialists or long distance monitoring of patients 32 or sharing patient specific information 11. However, the orderlies do not require monitoring or awareness of specific co-workers or patients. They focus on equipment, patients or co-workers relevant for their current task, but do not necessarily need a specialist's knowledge. Furthermore, the orderlies are focused on other potential tasks that can be scheduled into their current task schedule, to enable an efficient flow.

The main point advocating for wearable computing in this domain is the potential to get the overview and notifications, with little effort required to switch back and forth from their task, to the task management. Furthermore, the orderly can keep patient contact while still being aware of the work going on at the hospital. One way to support this is trough a glanceable device; thereby the user (orderly) can determine the notification's content without the patient feeling set aside. The wearable devices could provide notifications in a subtle, yet noticeable manner for the intended receiver. Whereas such notification provision, is more challenging with handheld smartphones, and examples of these challenges have been presented. Furthermore, as described, the glanceable awareness can be used to be aware of the task plans. This would support the orderlies in their coordination of tasks, which happens regularly and in an ad-hoc manner.

Another theme was the orderlies' perceptions of the wearable prototypes as socially acceptable and was highlighted as a feature, because they were perceived more as being a work tool than the handheld devices. Since what is considered socially acceptable today may change in 5 to 10 years as wearable devices will be more used in the leisure domain this might change the perception of these as being work tools.
In light of this, research should be continued to design novel wearable designs, for a broad range of hospital service work.

The data is of course constrained within the study's setting, since the scenarios were constructed and did not include any group coordination or patient contact. Yet the orderlies all agreed that the scenarios were representative of their work and interaction with PosLogistics. The study with its small sample of expert domain users only included 4 orderlies. However, the qualitative methods applied enabled both detailed investigations into the interaction as well as discussions of their current work practice. Moreover, the orderlies' initial impressions were very promising and they were positive towards including the wearable prototypes in their work. Based on this, the orderlies find the wearable devices usable to support their work, to a large extent even more than the handheld smartphone, if the issues with wearability and hygiene can be addressed with improved designs. The orderlies had several proposals on how this could be achieved by making it a more natural extension of the body, e.g., by using a sleeve with a smaller and more slim screen.

\section{CONCLUSION}

This paper has reported on a case study of future designs of wearable concepts for the hospital service domain. We have extended the current evidence to motivate wearable computing as useful to support such work. The themes for motivating includes easy information access, which would enable the orderlies to easier make the switch from focusing on a task, to report back to a task management system. This would allow the orderlies to be even more efficient, as they can glance at the current tasks and schedule their tasks more efficiently by having an overview. This case study has also highlighted that wearable devices can potentially be perceived as being a work tool by the workers even more so than mobile phones. This is important in many domains involving co-workers of different work groups, in our case study the orderlies and the clinical staff (nurses, doctors etc.). Furthermore, it is not only important for co-workers but also the patients, which might see the workers interacting with these devices that they are perceived as work tools. The investigations highlighted that the currently used device, a regular handheld smartphone, has introduced several work-arounds, because of the potential social perception of using such a device in a work setting.

While this study focuses on hospital service work, the issues with the interaction and use of handheld devices extend into other professional domains; for instance, supporting the ability to get an overview of the tasks at a facility while working can be a concern in other logistic domains. These concerns present important issues that can hinder the use of wearable devices in professional domains. Thus, to achieve the promising potentials of wearable computers the challenges presented here need to be addressed by future research in order to guide future designs of wearable devices.

\section{ACKNOWLEDGMENTS}

The authors would like to thank the orderlies at HO1, $\mathrm{HO} 2$ and Systematic for their help and valuable feedback and Nervo Xavier Verdezoto for his valuable comments. The authors acknowledge the support granted by the Danish Advanced Technology Foundation under J.nr. 076-2011-3. 


\section{REFERENCES}

[1] K. Adamer, D. Bannach, T. Klug, P. Lukowicz, M. L. Sbodio, M. Tresman, A. Zinnen, and T. Ziegert. Developing a wearable assistant for hospital ward rounds: an experience report. In Proc. IOT. Springer, 2008.

[2] D. L. Ashbrook, J. R. Clawson, K. Lyons, T. E. Starner, and N. Patel. Quickdraw: the impact of mobility and on-body placement on device access time. In Proc. CHI. ACM, 2008.

[3] J. Bardram and C. Bossen. Mobility work: The spatial dimension of collaboration at a hospital. In Proc. $C S C W$. Springer, 2005.

[4] J. E. Bardram and T. R. Hansen. Why the plan doesn't hold: A study of situated planning, articulation and coordination work in a surgical ward. In Proc. $C S C W . \mathrm{ACM}, 2010$.

[5] J. E. Bardram, T. R. Hansen, and M. Soegaard. AwareMedia: a shared interactive display supporting social, temporal, and spatial awareness in surgery. In Proc. CSCW. ACM, 2006.

[6] G. Bieber, T. Kirste, and B. Urban. Ambient interaction by smart watches. In PETRA. ACM, 2012.

[7] V. Braun and V. Clarke. Using thematic analysis in psychology. Qualitative Research in Psychology, $3(2): 77-101,2006$

[8] J. Brooke. Sus-a quick and dirty usability scale. Usability evaluation in industry, 189, 1996.

[9] B. Brown, M. McGregor, and E. Laurier. iPhone in vivo: video analysis of mobile device use. In Proc. CHI. ACM, 2013.

[10] D. Fallman. Wear, point, and tilt: designing support for mobile service and maintenance in industrial settings. In Proc. DIS. ACM, 2002.

[11] G. Fitzpatrick and G. Ellingsen. A review of 25 years of cscw research in healthcare: Contributions, challenges and future agendas. Computer Supported Cooperative Work, 22(4-6):609-665, 2013.

[12] F. Gemperle, F. Gemperle, C. Kasabach, C. Kasabach, J. Stivoric, J. Stivoric, M. Bauer, M. Bauer, R. Martin, and R. Martin. Design for wearability. In Proc. ISWC, 1998.

[13] V. M. González, M. E. Tentori, E. B. Morán, J. Favela, and A. I. Martínez. Understanding mobile work in a distributed information space: Implications for the design of ubicomp technology. In Proc. CLIHC. ACM, 2005.

[14] R. Hansson, P. Ljungstrand, and J. Redström. Subtle and public notification cues for mobile devices. In Proc. UbiComp. Springer, 2001.

[15] C. Harrison, B. Y. Lim, A. Shick, and S. E. Hudson. Where to locate wearable displays?: reaction time performance of visual alerts from tip to toe. In Proc. CHI, 2009.

[16] M. B. Kjærgaard, M. V. Krarup, A. Stisen, T. S. Prentow, H. Blunck, K. Grønbæk, and C. S. Jensen. Indoor positioning using wi-fi-how well is the problem understood? In IPIN, 2013.

[17] J. F. Knight, D. Deen-Williams, T. N. Arvanitis, C. Baber, S. Sotiriou, S. Anastopoulou, and M. Gargalakos. Assessing the Wearability of Wearable Computers. In Proc. ISWC, 2006.
[18] R. Ling. "'One Can Talk about Common Manners!": The Use of Mobile Telephones in Inappropriate Situations'. Themes in mobile telephony Final Report of the COST 248 Home and Work group., 1997.

[19] J. Liu, Z. Wang, L. Zhong, J. Wickramasuriya, and V. Vasudevan. uWave: Accelerometer-based personalized gesture recognition and its applications. In Proc. PerCom, 2009.

[20] T. Prentow, H. Blunck, K. Grønbæk, and M. Kjærgaard. Estimating common pedestrian routes through indoor path networks using position traces. In Proc. MDM, 2014.

[21] H. P. Profita, J. Clawson, S. Gilliland, C. Zeagler, T. Starner, J. Budd, and E. Y.-L. Do. Don't mind me touching my wrist: a case study of interacting with on-body technology in public. In Proc. ISWC. ACM, 2013.

[22] M. Reddy and P. Dourish. A finger on the pulse: Temporal rhythms and information seeking in medical work. In Proc. CSCW. ACM, 2002.

[23] M. J. Sammon, L. S. Brotman, E. Peebles, and D. D. Seligmann. MACCS: enabling communications for mobile workers within healthcare environments. In Proc. MobileHCI. ACM, 2006.

[24] J. Scholl, P. Hasvold, E. Henriksen, and G. Ellingsen. Managing Communication Availability and Interruptions: a Study of Mobile Communication in an Oncology Department. In Pervasive computing, 2007.

[25] J. Siegel and M. Bauer. A field usability evaluation of a wearable system. In Proc. ISWC, 1997.

[26] M. B. Skov and R. T. Høegh. Supporting information access in a hospital ward by a context-aware mobile electronic patient record. Personal and Ubiquitous Computing, 10(4):205-214, 2005.

[27] T. Starner. The challenges of wearable computing: Part 2. Micro, IEEE, 21(4):54-67, 2001.

[28] M. Tentori and J. Favela. Activity-aware computing for healthcare. Pervasive Computing, 7(2):51-57, 2008

[29] M. Tentori, G. R. Hayes, and M. Reddy. Pervasive computing for hospital, chronic, and preventive care. Foundations and Trends in Human-Computer Interaction, 5(1):1-95, 2012.

[30] F. Ulger, S. Esen, A. Dilek, K. Yanik, M. Gunaydin, and H. Leblebicioglu. Are we aware how contaminated our mobile phones with nosocomial pathogens? Annals of Clinical Microbiology and Antimicrobials, 8(1):7, 2009.

[31] K. A. Weaver, H. Baumann, T. Starner, H. Iben, and M. Lawo. An empirical task analysis of warehouse order picking using head-mounted displays. In Proc. CHI. ACM, 2010.

[32] P. Weller, L. Rakhmetova, Q. Ma, and G. Mandersloot. Evaluation of a wearable computer system for telemonitoring in a critical environment. Personal and Ubiquitous Computing, 14(1), 2010.

[33] L. Wu, J. Cirimele, J. Bassen, K. Leach, S. Card, L. Chu, K. Harrison, and S. Klemmer. Head-mounted and multi-surface displays support emergency medical teams. In Proc. CSCW, 2013. 